\title{
Optimal PMU Configuration Based on Improving Teaching-learning-based Optimization Algorithm
}

\author{
Xiaodong $\mathrm{Li}^{1{ }^{1 *}}$ and Mingyu Jing ${ }^{2}$ \\ ${ }^{1}$ School of Electrical Engineering \& Information Engineering, Lanzhou University of Technology, Lanzhou 730050, China \\ ${ }^{2}$ State Grid Gansu Electric Power Company Qingyang Power Supply Company, Qingyang 745000, China \\ ${ }^{*}$ Corresponding author
}

\begin{abstract}
Taking the full network observability of power system operation state and the least number of phasor measurement units(PMU) as objective, this paper proposes an optimal PMU placement algorithm based on an improving teaching-learning-based optimization algorithm(ITLBO). Phasor measurement units can measure bus voltage phasors, combined with rapid topological observable analysis method, the power system can be fully observable. And then proposes the improving teaching-learning optimization algorithm to solve the optimal configuration problems, to achieve the global optimum, finally get optimal configuration scheme of measuring points. Finally harmonic state estimation is carried out on the basis of this, and programme in matlab and compare with the binary teachinglearning optimization algorithm verify its validity of the proposed method.
\end{abstract}

Keywords-teaching-learning-based optimization algorithm; phasor measurement units; Optimal PMU configuration; harmonic state estimation

\section{INTRODUCTION}

This With the wide application of the global positioning system (GPS), PMU was used in the power system. The PMU can measure the phase of the bus voltage compared with a traditional monitoring and acquisition system that can only obtain node voltage amplitude, active power, and reactive power ${ }^{[1]}$. How to configure PMU has become a common concern of power companies. The PMU can directly measure the amplitude and phase angle of the node voltage vector, and can also measure the current of the branch connected to the node to achieve dynamic monitoring of the power grid ${ }^{[2]}$. In order to obtain the complete key parameters of the power grid, the configuration of the PMU must make the system visible. If all buses on the entire network are configured with PMU, the entire system is fully observable without any iterative calculations.

In the research of harmonic state estimation in recent years, the main ideas for research on the optimization configuration of PMU are: in order to improve the observability of the harmonic state estimation, through increasing measurement point to get the optimal configuration of PMU on the basis of the existing measuring equipment, and reasonably placing gauge point to get the optimal configuration of PMU when the number of PMU configurated is certain. Considering the configuration of the measurement substation and measurement channel comprehensively, the optimal configuration of the PMU that guarantees a considerable harmonic state of the system and considers the estimation accuracy ${ }^{[2-6]}$. The optimal configuration of the PMU can be obtained by applying the Binary PSO Algorithm ${ }^{[7]}$. a new genetic algorithm based method for optimal placement of phasor measurement units (PMUs) considering observability and security issues is proposed $^{[8]}$. a 0-1 semidefinite programming (SDP) approach to solve the problem of optimal placement of phasor measurement units (PMUs), considering the existence of conventional measurements and zero injections as well as the impact of PMU channel limits ${ }^{[9]}$. However, since the mathematical model of the PMU optimization configuration contains the estimation error of the harmonic state, once the algorithm is solved, the harmonic state estimation needs to be performed once. In addition, according to the difference of each measurement, the method of harmonic state estimation is also different, which not only has a large amount of calculation but also has a complicated solution ${ }^{[10]}$.

\section{POWER SySTEM OBSERVABILITY}

\section{A. Algebraic Observability}

The observability of power system can be analyzed by numerical method or network topology method. The linear measurement model of a power system with $N$ node number and $m$ dimensional measuring vector $m$ can be expressed as

$$
z=H x+v
$$

where, $Z$ is the $m$ dimensional measurement vector; $H$ is $2 N-1$ dimensional measurement the jacobian matrix; $X$ is the $2 N-1$ dimensional voltage state vector; $v$ is $m$ dimensional measurement noise vectors.

The theoretical base of linear measurement model of algebraic observability can be directly solved, which means that if $\operatorname{rank}(H)=2 N-1$, namely the rank of $H$ is full, the system can be considered to be algebraic observable. The closer the maximum and minimum singular value is, the smaller the condition number of the corresponding measurement matrix and the higher the observability of the system. $\operatorname{Cond}(A)$ is a measure index of its near singularity. In order to improve the observability of the system, it should be reduced as much as possible. 


\section{B. Topological Observability}

The According to graph theory knowledge, a power system can be seen as the figure $G=(V, E)$ made up of $N$ nodes and $b$ edges, $V$ represents the nodes sets of the figure, $E$ represents the edges sets of the figure, respectively corresponding to a collection of bus and branch of system. The measurement network constitutes a measurement subgraph, that $V^{\prime} \subseteq V, E^{\prime} \subseteq E$ is naturally satisfied. If the relationship between the measurement subgraph $G^{\prime}$ and the graph $G$ is satisfied with $V \subseteq V^{\prime}$, that is, all nodes of the graph are included in the subnetwork diagram, and the system is topological observable.

\section{MATHEMATICAL MOdEl OF PMU CONFIGURATION}

\section{A. Definition of Optimal PMU Configuration Problem}

For a $n$ node system, define:

$$
\begin{array}{ll}
\min & m=\sum_{i=1}^{n} x_{i} \\
\text { s.t } \quad \mathrm{U}_{i}=1
\end{array}
$$

where $m$ represents the number of PMU; $U_{i}$ means that bus $i$ can be observed; $i=1,2, \ldots, N ; N$ represents the number of bus bars. The dimension of the solution of the optimization problem is equal to the number of bus bars of the power system, and the value of each dimension is binary coded, that is, the value of each dimension can only be 0 or 1 . When the PMU is installed in a busbar, the corresponding position is Figure I.

\begin{tabular}{|l|l|l|l|l|l|l|}
1 & 2 & 3 & $\cdots$ & $\mathrm{N}-2$ & $\mathrm{~N}-1$ & $\mathrm{~N}$ \\
\hline 0 & 1 & 0 & $\cdots$ & 1 & 0 & 1 \\
\hline
\end{tabular}

\section{FIGURE I. PARTICLE CODING DIAGRAM}

In the observability analysis of power system, it is necessary to determine whether the network can be observed and how many times a bus is observed, so the observation redundancy is defined:

$$
R=\sum_{i=1}^{N} V_{\text {obser }}(i)-N
$$

where $N$ is total the number of busbars in the network. When the system satisfies that the total number of visible PMU configurations is the same, it is necessary to further investigate the redundancy index.

\section{B. PMU Configuration Rules}

When a power plant or transformer substation is configured with PMU, the device can measure the voltage phasor of the bus and all the branch currents phasors connected to it. According to kirchhoff's law and ohm's law, the conclusions are as follows.

Conclusion 1: if the node $i$ is equipped with PMU, then the bus voltage phasor of the power plant or transformer substation corresponding to node $i$ can be measured, and the node $i$ can be observed.

Conclusion 2: if the node $i$ is placed with PMU, then all of busbar voltage phasor of the power plants or transformer substations conected to the node $i$ can be indirectly measured, because the outgoing current current corresponding to the node can be directly measured by PMU, the busbar voltage phasors of the contralateral power plant or transformer substation can obtain according to the ohm's law and the circuit parameters. Therefore, adjacent nodes can also be observed.

Conclusion 3: there are some circuits which line currents are known such as generator outlet in the power system. when a node can be observed, the opposite nodes are also observable.

Conclusions 4: in power system, there are a large number of nodes with zero power injection, and if a node and nodes with the numder of $m$ are linked together, if nodes with the numder of $m$-1among $m$ nodes can be observed, thus the $m t h$ node be observed.

\section{Optimization CONFIgURATION OF THE PMU BY IMPROVING TLBO}

\section{A. Teaching-learning Optimization Algorithm}

The TLBO algorithm is a new swarm intelligence optimization algorithm, which has not been used to optimize the PMU configuration of power system in China. The principle is to simulate the teaching process of teachers and the learning process of students. The purpose is to improve students' academic performance through "teaching" of teachers and "learning" between students. The advantages of TLBO are less algorithm parameters, simple algorithm, easy to understand, fast operation speed, high precision and strong convergence. The basic idea of TLBO is to simulate study way with class as the unit. improvement of academic records of students in the class need guidance of "teaching", at the same time, through "learning" between each other to promote mastery of knowledge. Obviously, the teachers and students amount to individuals in evolutionary algorithms, while the teacher is the best individuals.

\section{B. PMU Optimization Step Based on Improved TLBO Algorithm}

1) Initialize the class. Each student in the class is randomly generated in the search space.

$$
X_{i}=X_{i}^{\min }+\operatorname{rand}\left(X_{i}^{\max }-X_{i}^{\min }\right)
$$


where $X_{i}^{\max }$ and $X_{i}^{\min }$ are the lower limit value and the upper limit value of the control variables respectively. rand is a random number ranging from 0 to 1 .

2) The "teaching" stage.

The teacher's duty is to improve the average grade of the whole class, and gradually raise the average grade of the class through the teacher's repeated efforts. At the "teach" stage, each student learns from the teacher by finding the difference between teacher's fitness and the student's average fitness. "Teach" according to the following formula.

$$
\begin{gathered}
M^{k}=\frac{\sum_{i=1}^{N p} X_{i}^{k}}{N p} \\
X(i, j)_{\text {difference }}^{k}=\operatorname{rand} \times\left(X_{T}(j)^{k}-t_{f} M_{T}(j)^{k}\right)
\end{gathered}
$$

where $X_{T}(j)^{k}(j=1,2, \ldots, d)$ represents the achievement value of subject $k$ of teacher in the $j$ th iteration. $M_{T}(j)^{k}$ indicates mean achievement value of subject $k$ of student in the $j$ th iteration in the class. $t_{f}$ is the teaching factor, which generate according to the following formula.

$$
t_{f}=\operatorname{round}[1+\operatorname{rand}(0,1)]
$$

Refer to the concept of the linear decreasing inertia weight in particle swarm algorithm to ameliorate teaching factor of teaching-learning-based optimization algorithm, so it can change adaptively in the light of iterations, which can not only converge rapidly at the beginning of the search and search accurately in the late search. After improvement, $t_{f}$ is generated by following formula.

$$
t_{f}=t_{f \max }-\frac{t_{f \max }-t_{f \min }}{\text { iter }_{\text {max }}} \text { iter }
$$

where $t_{f \max }$ and $t_{f \text { min }}$ are respectively the maximum and minimum values of $t_{f}$. ${ }^{\text {iter }} r_{\text {max }}$ is the maximum iterations and iter is nowaday iterations. update solution according to the following formula.

$$
\begin{aligned}
& X(i, j)_{\text {new }}^{k+1}=X(i, j)_{\text {old }}^{k}+X(i, j)_{\text {difference }}^{k} \\
& i=1,2, \ldots, N p, j=1,2, \ldots, d
\end{aligned}
$$

3) "learning" stage.

At this stage, each student in the class randomly selects one other student as the learning object in the class. If other students are better than themselves, they learn from other students. The formula is as follows.

$$
\begin{gathered}
X_{i}^{\text {new }}=\left\{\begin{array}{c}
X_{i}^{\text {old }}+\operatorname{rand} \times\left(X_{m}-X_{i}^{\text {old }}\right), f\left(X_{m}\right)<f\left(X_{i}^{\text {old }}\right) \\
X_{i}^{\text {old }}+\operatorname{rand} \times\left(X_{i}^{\text {old }}-X_{m}\right), f\left(X_{m}\right)>f\left(X_{i}^{\text {old }}\right)
\end{array}\right. \\
m=\text { ceil }(N p \times \text { rand }), m \neq i
\end{gathered}
$$

where $X_{m}=\left(X_{m 1} X_{m 2}, \mathrm{~K}, X_{m d}\right), f\left(X_{m}\right)$ is the fitness value of the $m$ th student.

Binary coding is introduced, in order to enable TLBO to solve the discrete optimization problem as optimal PMU configuration in the power system. In the teaching-learningbased optimization algorithm of binary coding, students per dimension can only take 0 or 1 . In order to satisfy this condition forever in the iterative process, the fuzzy function $\operatorname{sig}(x)$ is introduced.

$$
X(i, j)_{\text {new }}^{k+1}= \begin{cases}0 & \mathrm{r} \geq \operatorname{Sig}\left(X(i, j)^{k}\right) \\ 1 & \mathrm{r} \leq \operatorname{Sig}\left(X(i, j)^{k}\right)\end{cases}
$$

where, $r$ is the random number between 0 and $1 . X(i, j)$ is limited to the set $\{0,1\}$. In the teaching-learning-based optimization algorithm of binary coding, Each dimensional component of a student takes 1 with the probability of $\operatorname{sig}\left(X(i, j)^{k}\right.$ and takes 0 with the probability of $1-\operatorname{sig}\left(X(i, j)^{k}\right.$.

\section{HaRmonic State Estimation Based on PMU OPTIMIZATION CONFIGURATION}

Harmonic measurement variables selected with harmonic state estimation are typically node voltage phasors, node injection harmonic currents, or harmonic current phasors of branch. Choice of state variables are node harmonic voltage phasors. According to the parameters of the power network to establish node harmonic admittance matrix, write harmonic measurement equations of state estimation.

When measurements are the harmonic currents of branches connected to a node, with the node harmonic voltage phasors as the state variables, the measurements equations are as follows:

$$
\dot{I}_{i}(h)=\left(Y_{i}+1 / Z_{i j}\right) \dot{U}_{i}(h)-\left(1 / Z_{i j}\right) \dot{U}_{j}(h)+\eta_{i}(h)
$$

where $\dot{I}_{i}(h)$ is measurement phasor of harmonic current flowing from node $i$ into the branch between node $i$ and $j \cdot \dot{U}_{i}(h)$ and $\dot{U}_{j}(h)$ are respectively state variables of 
hth harmonic voltage corresponding to node $i$ and node $j . \eta_{i}(h)$ is harmonic measurement error of node $i$ under the action of $h$ th harmonic. The branch between $i$ and node $j$ is equivalent to the $\Pi$ equivalent circuit, $Y_{i}$ is the ground branch admittance matrix and $Z_{i j}$ is the branch impedance matrix.

When the equivalent measurements are nodal injection current phasors, the measurement equations are as follows:

$$
\dot{I}_{i}(h)=\sum_{j=1}^{n} Y_{i j}(h) \dot{U}_{j}+\eta_{i}(h)
$$

where $Y_{i j}$ is the block admittance matrix corresponding to node $i$ and node $j$ in the node admittance matrix.

When the equivalent measurements are the node harmonic voltage phasors, the measurements equations are as follows:

$$
U_{i}(h)=1 \times U_{i}(h)+\eta_{i}(h)
$$

Write measurements equations according to the known measurements, the influence of measurements errors are ignored. The mathematical model of harmonic state estimation can be expressed as:

$$
Z=H X
$$

where $Z$ are known measurable vectors, $X$ are the state vectors to be estimated, and $H$ is the measurement matrix for the relationship between the two.

In this paper, the classical least square method is used to estimate the harmonic voltage phasors, in order to verify the validity of the proposed algorithm applying to optimal configuration of PMU.

\section{ANALYSIS OF EXAMPLES}

Taking the New England 39 bus system as an example, the system simulation and analysis are carried out, The structure of the IEEE-39 node standard test system is shown in Figure II, which consists of 39 bus nodes and 10 generator nodes, and contains 12 zero power injection nodes, including 5, 6, 9, 15, 17, 20, 21, 23, 24, 25, 28, 29, etc. In the PSCAD(Power System Computer Aided), a 39 node system was set up to extract the required measurement data and line parameters. The program of improving teaching-learning-based optimization algorithm, observability analysis and optimization configuration of PMU measurement and harmonic state estimation using classical least square method was developed in matlab. The number of students is 100 and the number of iterations is 200. The simulation results of the IEEE-39 node standard test system are as follows:

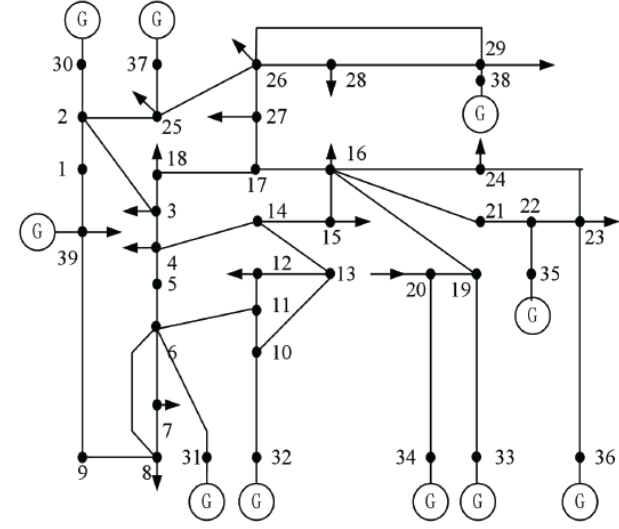

\section{FIGURE II. SIMPLIFIED DIAGRAM OF NEW ENGLAND 39 BUS} SYSTEM

It can be seen from Figure III that the binary teachinglearning-based optimization algorithm introducing adaptive teaching factors has obvious improvement in convergence speed compared with ordinary binary teaching-learning-based optimization algorithm.The ITLBO (improving teachinglearning-based optimization algorithm) can obtain more feasible solutions, and the comparison results are shown in Table I. For the measurement configuration scheme of the greater redundancy, when the PMU fails, it can still maintain most of the nodes, or even all observable.

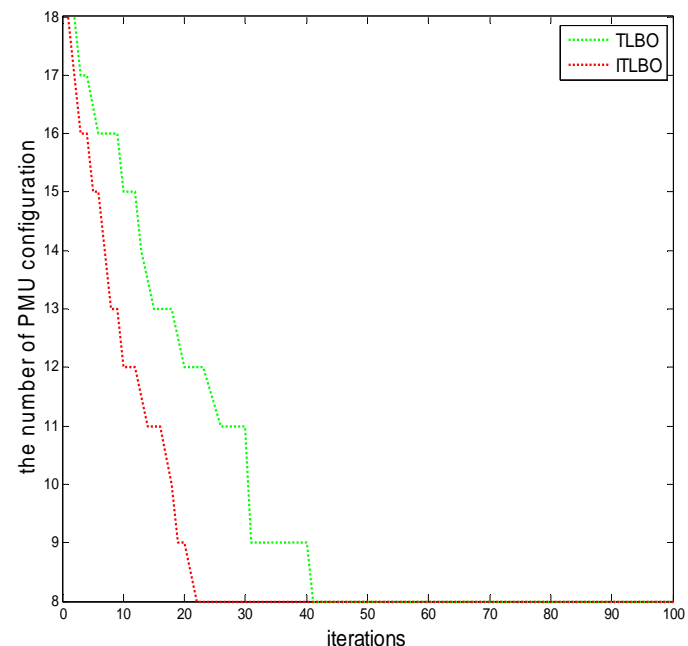

FIGURE III. COMPARISON OF ITLBO AND TLBO FOR NEW ENGLAND 39-BUS SYSTEM

\begin{tabular}{|c|c|c|c|c|c|c|c|c|c|}
\hline $\mathbf{N}$ & $\mathbf{R}$ & \multicolumn{8}{|c|}{ PMU installation node } \\
\hline \multirow{8}{*}{8} & \multirow{2}{*}{5} & 3 & 8 & 10 & 16 & 20 & 23 & 25 & 29 \\
\hline & & 3 & 8 & 12 & 16 & 20 & 23 & 25 & 29 \\
\hline & \multirow{2}{*}{4} & 3 & 8 & 10 & 16 & 23 & 25 & 29 & 34 \\
\hline & & 8 & 13 & 16 & 18 & 20 & 23 & 25 & 29 \\
\hline & \multirow{3}{*}{3} & 3 & 8 & 10 & 16 & 20 & 23 & 29 & 37 \\
\hline & & 3 & 8 & 12 & 16 & 20 & 23 & 29 & 37 \\
\hline & & 8 & 13 & 16 & 18 & 23 & 25 & 29 & 34 \\
\hline & 1 & 3 & 8 & 13 & 16 & 23 & 29 & 34 & 37 \\
\hline
\end{tabular}

TABLE I. OPTIMAL PLACEMENT SCHEME OF PMU FOR NEW ENGLAND 39-BUS SYSTEM 
Applying optimal PMU configuration scheme above with redundancy equal to 5 , 3th, 5th, 7th harmonic currents were injected into the node 5,8 and 19 respectively, and the harmonic state estimation was performed. It can be seen that all the absolute value of amplitude errors of the harmonic voltage phasors are less than 5\%, and that of phase angle errors of the harmonic voltage phasors also meet the related requirements of accuracy (See Figure IV-V).

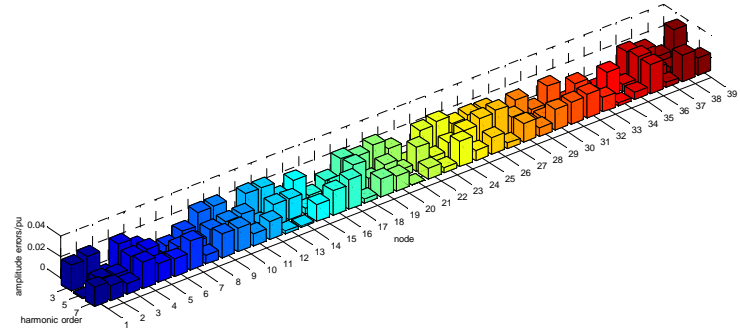

FIGURE IV. ESTIMATED ERROR OF THE MAGNITUDE OF THE NODE HARMONIC VOLTAGE

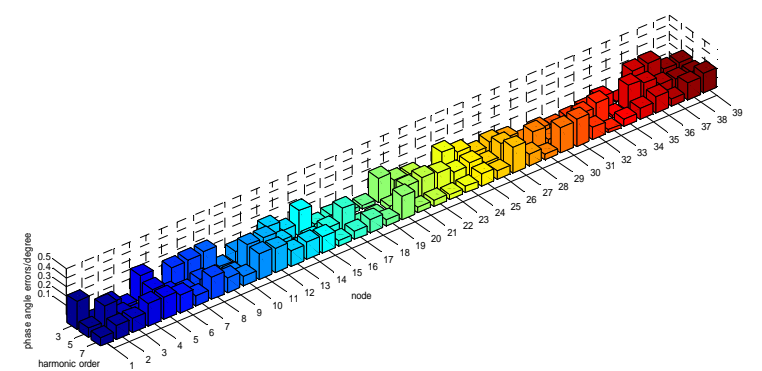

FIGURE V. ESTIMATED ERROR OF THE PHASE OF THE NODE HARMONIC VOLTAGE

\section{CONCLUSION}

In this paper, the adaptive teaching factor is introduced to improve the TLBO algorithm for the first time, Binary coding realizes the discretization of teaching-learning-based optimization algorithm, which can effectively solve the problem of optimization configuration of PMU configuration, for minimum number of measured points and maximum redundancy. The proposed algorithm can achieve the goal of the minimum number of PMU configuration under the premise that the system is fully observable, and provide the redundancy as the contrast parameter. The feasibility of the algorithm is proved by the IEEE-39 standard power system. At the same time, the method can provide a variety of PMU configuration schemes for providing more options for practical applications. Finally, the harmonic state is estimated by using measurement configuration scheme of the redundancy equanl to 5 . The accuracy of harmonic state estimation is found to be within the allowable range by calculating absolute errors of the amplitude and phase angle of the estimated harmonic voltage phasors, which verifies the validity of the proposed PMU configuration method.

\section{ACKNOWLEDGMENT}

The authors were grateful to project supported by Gansu natural science foundation(1308RJZA245), name of which is the diffusion mechanism of harmonic in complex power network. Wei CHEN, my supervisor thanks guidance to my thesis.

\section{REFERENCES}

[1] S. S. Noureen, V. Roy and S. B. Bayne, "Phasor measurement unit integration: A review on optimal PMU placement methods in power system," 2017 IEEE Region 10 Humanitarian Technology Conference (R10-HTC), Dhaka, 2017, pp. 328-332.

[2] P. K. Ghosh, S. Chatterjee and B. K. Saha Roy, "Optimal PMU placement solution: graph theory and MCDM-based approach," in IET Generation, Transmission \& Distribution, vol. 11, no. 13, pp. 3371-3380, 972017.

[3] Zhao hui, Zhao shaohua, Wang hongjun, Yue youjun, "Optimization of PMU configuration based on 0-1 programming iteration method to improve the accuracy of mixed state estimation," in Electrotechnical Application, vol. 36,no. 5,pp.80-84, 2017.

[4] Xu Yan, Ying Luman, Wang Zengping, "Staged Phasor Measurement Unit Placement Algorithm Based on Theory of Maximum Tree," in Transactions of China Electrotechnical Society, vol. 31,no. 4,pp. 155162, February 2016.

[5] Bao Wei, Jiang Xuedong, Chen Liyue, Lu ming, Guo ruipeng, "a 0-1 linear programming approach to optimal phasor measurement units placement considering maximized system observability redundancy," in Power System Technology, vol. 38,no. 8, pp. 2052-2056, August 2014.

[6] Mao Yi, Lv Feipeng, "Optimal PMU placement method based on evolutionary game algorithm," in Electric Power Automation Equipment, vol. 37,no. 10,pp. 184-188, October 2017.

[7] N. H. A. Rahman and A. F. Zobaa, "Integrated Mutation Strategy With Modified Binary PSO Algorithm for Optimal PMUs Placement," in IEEE Transactions on Industrial Informatics, vol. 13, no. 6, pp. 31243133, December 2017.

[8] H. H. Müller and C. A. Castro, "Genetic algorithm-based phasor measurement unit placement method considering observability and security criteria," in IET Generation, Transmission \& Distribution, vol. 10, no. 1, pp. 270-280, July 2016.

[9] N. M. Manousakis and G. N. Korres, "Optimal PMU Placement for Numerical Observability Considering Fixed Channel Capacity-A Semidefinite Programming Approach," in IEEE Transactions on Power Systems, vol. 31, no. 4, pp. 3328-3329, July 2016.

[10] Zhang Siwei, "Research on Power System Harmonic State Estimation Based on Optimal PMU Placement and Measurement Information," Heibei: North China Electric Power University, 2013.

[11] T. Niknam, F. Golestaneh and M. S. Sadeghi, "\$theta-Multiobjective Teaching-Learning-Based Optimization for Dynamic Economic Emission Dispatch," in IEEE Systems Journal, vol. 6, no. 2, pp. 341-352, June 2012.

[12] Rao R V, Savsani V J, Vakharia D P, "Teaching-learning-based optimization: a novel method for constrained mechanical design optimization problems," in Computer-Aided Design, vol. 43, no. 3,pp. 303-315, March 2011. 